ihres Auftretens ein genaucres Eingehen auf verborgene Magen-Darmsymptome noch verhindert.

2. Gruppe: In dieser Gruppe lassen sich Störungen zusammen. fassen, bei denen dic Magen-Darmerscheinungen den vorhandenen cndokrinen Krankheitszeichen nicht ohne weiteres unterzuordnen sind, sonderı dic vielleicht mit ihnen ciner dritten, übergcordneten Ursache zuzuschreiben sind. Es ist dies besonderș dats Gebiet der Magellund Darmneurosen, über dic der Kricg nicht zıl Interschätzendes Material geliefert hat. Dic Bcobachtungen von $\mathrm{C}$ a ro (3) und nament lich von $\mathrm{H}$. Strauß (4) habcn gezeigt. daß Magenncurosen augenschcinlich glcichzeitig mit ciner Thyrcotoxikose in einem Milicu vor kommen können. das der Ncuropathic nahestcht. Wenigstciıs komm Strau $B$ ju der Ansicht daß die Schilddrüsensymptome nicht ohne weitercs für die Magenne:ırose verantwortlich gemacht werdell können. wenn auch in der Ueberfunktiọn dieser Drüse sẹthst wieder Faktoren gegeben sind. dic den allgemeinen Status nervosus zul steigern ver mögell. Die Erfahrung zcigt. daß unter diesc Gruppe auch wohl manche Fälle von Maigengeschüüren fallen. bzw. solche Fälle, be denen es klinisch oft unmöglich ist festzustcflen, ob es sich hier noch um einc rein funktionelle motorisch-sekretorische Neurose oder schon $\mathrm{um}$ cin fertiges Ulkiıs handelt Dahin gehören manclıe Fälle von $\mathrm{Oh}$ stipation, bei denen der Wechsel zwischen spastischen und Erschlaf fungszuständen des Darms eiıen Tonuswëchsel im sympathischen und autonomen Nervensystem voraussetzen läßt. der einc endokrinc Be. teiligung. auch wenn sic nicht schärfer zu präzisicren ist, zum mindesten als determinierenden Faktor aufweisen kann.

3. Gruppe: Hierhinein wären diejenigen Krankhcitszustände zı (Direktor: Prof. Volhard.)

\section{Chronische Kolitiden mit sekundären inkretorischen Störungen und ihre Bedeutung fuir die Konstitution.}

\author{
Von Priv.-Doz. Dr. L. R. Grote.
}

Durch dic Verhandlungen der letzten. Tagung für VerdauungsInud Sioffuechselkrankhciten in Homburg ist die Frage der Beziehungen zwischen den Intestinalerkrankungen und den inkretorischen Drüsen in Flıß gekommen. Es war zweifellos sehr dankenswert. dicsen Gegenstand vor einem größcrenl Forum zur Besprechung zu stellen. weil dadurch manche schwebenden Fragen ciner Klärung z.l1. geführt werden können. Das in dicsen Verhandlungen zutagege. förderte Material war allerdings keineswegs so gcartet, daß ein abgeschlossenes Bild des ganzen Problems resultierte. Vielmelır zeigte es sich daß wir im wesentlichen noch mit Ansätzen und Versuchen auf diesem Gebiet zu rechnen haben. aus denen sich die Richtlinien für einc systematische Bearbeitung der ganzen Fragen erst noch ergeben. So sind im besonderen die Beziehungen, die die Intestinalorgane im engeren Sinne d. h. Magen und Darm, zum cndokrincn System aufweisen. klinisch noch zum großen Teil wenig beaditct. Auch das von Bicdl erstattete Referat ließ gerade in dicser Beziehung noch viele Fragen offen. während es die diagnostischen Mög. lichkeiten für andere Erkrankungen (Stoffwechselstörungen. Wachstums. störungen u. a.) ungleich deutlicher zur Darstellung bringen konnte.

Bei der zunehmenden Erkenntris auf dem Gebicte inkretorischer Störungen ist es aber mit Sicherheit zu erwarten, d:a dieses System, das zu allen anderen Organsystemen des Körpers und ihren wechselseitigen Beziehungen so enge Bindung aufwcist. bezüglich der MagenDarm-Pathologie keine Ausnahme machen wird. Im allgemeinen ist man doch schon so weit gekoninicn, daß man Veränderungen des endokrinen Systems etwa in folgender Weise zu Erkrankungen des Magen-Darmkanals in Beziehung setzen kann. Die dahin gehörigen Möglichkciten lassen sich in 3 Gruppen zusammenfassen:

1. Gruppe: Hicrher gchören diejenigen Störungen der MagenDarmfunktion; die als unmittelbarer Ausdruck einer primären Er. krankung inkretorischer Drüsen aufzufassen sind. Sckretionsstörungen des Magens bei der Basedowschen Krankheit sind bckannt, im großen und ganzen scheinen sie. wenngleich dilrüber widersprechende Angaben der Literatur z.u finden sind, z.u einer Depression der sekretorischen Tätigkeit der Magenschlcimhallt zıı führen. Es fchIt nicht. an Beobachtungen von Superadizität urid Supersckretionen. doch findet die Mchrzahl der Autoren ein Herabgehen der Magensaftabscheidungr. Dic Darmstörungen der Basedou'schen Erkrankıng sind bösartige Komplikationen. cbenso bekannt als ge. fürchtet. Die oft unstillbaren Durchfälle. dic gelegentlich einc ganz besondere Störung der Fettverdauung. sci es durch unmittelbaren Pankreassaftausfall. sei es durch Resorptionsstörung. aufwcisen. köninen das Bild so beherrschen, daß in cinzelnen Fällen der mehr oder weiniger intuitive therapeutische Versuch der Schilddrüsenentfernung (Kolb [1]. Curschmann [2]) die eigentliche Natur der Darmerkrankung aufklärte und sic bescitigtc. Bei der Addisonschen Krankheit finden wir ähnliche, wenn auch in der Regel nicht so sehr schwere Ausfallserscheinungen der Magen- und Darmtätigkeit. In spätercn Stadien besonders ist Achylie des Magens. nicht ohnc Ausnahme, dic Regel: Durchfälle, die häufig unter dem Zcichen gestörter Fettresorption einsetzen (Adolf Schmidt). können gegen das Ende hin sich żu organischen enteritischen. Krankhcitsbildern stcigern. Bei der Akromegalic scheint dic Obstipation die Darmtätigkeit zu beherrschen, aber dic sonstigen Verhältnisse ist nicht viel bekannt. Das Gleiche gilt für dic übrigen Krankheitsbilder, dic man mehr oder weniger umschrieben auf die vorwicgende Störung einer inkretorischen Drïsc zurückfiihrt. Bei manchen der in Bctricht kommenden Erkrankungren. so der pluriglandulären Insuffizienz. hat wohl dic relative Seltenheit

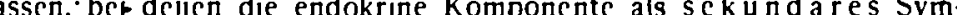
ptom auftritt. Eine Unterteilung ist möglich in solche, bei dencn

a) durch unmittelbare Fortleitung eines organischen Prozesses eille inkretorische Drüse geschädjgt $u$ ird. Das ist det Fall bei allen chronischen Enteritiden. dencn sich cinc sckundäre Pankrcatitis aufpfropft, in deren Gefolge sekretorische und inkretorische Störungen auftreten 'können (alimentäre Glukosuric).

In der 2. Untergruppe möchte ich

b) diejeñigen Fälle vercinigen. bei denen dieser Zusammenhang ein funktioneller ist. Es kinn sich in dieser Untergruppe sowohl um endokrinc Drüsen handeln. die dem Darm anatomisch unmittelbar nahestehen (Pankreashypochvlie oluric anatomische. Veränderung be gleichzeitiger Achylie oder Sıbazidität des Magens, funktionelle Pari kreasstörungen bei chronischen Dyspenșien), oder auch um soiche. die denı Darmkanal anatomisch fernstefen. wie die Schilddrïse.

Gerade diese Ictzte Untergruppe scheint cinc besondere Beact tung in der Klinik noch uicht gefunden zu haben, die ihr aber. nich nur aus unmittelharen therapeutiscticn Rücksichten licrans, doch zukommell dürfte. In Adolf $\mathrm{Schmidts}$, Klinik der Darmkrankheifon" lindet man über diese Zustände nur Andeutungen. und auch die übrige l.iteratur nimmt bis heute wenig Notiz davon ${ }^{1}$ ). und doch sind woh diesc sekundären funktionellen inkretoriscticn Störungen nicht ohne Belang.

Bei einem gröReren Material von chronischen Kolitiden dic als Rückständc durchgemachter Duscntcrien aufzufassen sind hathe ich die Beobachtung einer zwcifcllos sckundär im Verlauf der organischen Darmerkrankung auftretenden thyreotoxischen Störung .nich ganz sclten machen können. Auf Prozentzalılen will ich mich ilich cinlassen, weil ich früher auf dicse Verlı̈̈ltnisse nicht geachtet habe Im Verlallf etwa des Ictzten halben Jahres habe ich bis ictzt 9 Fäll sckundärer Thyreotoxikose bei nostdvsenterischer Kolitis gefunden. Als Bcjspicl greife ich aus den Krankengeschichten folgende heraus: H. K.. 33 Jahre, Feldwebel, Eltern gesund, in der Verwandtschaft an geblich keine erblichen Krankheiten. kam im August 1914 ins Feld. im Mai 1915 in Galizien Ruhrerkrankung, in ambulanter Bchandlimg gebessert. Im luli 1916 Wicderholung der Durchfällc, dic vicl Schleim und Blut enthieltell, nach kumer I.azarettbehandlung gebessert. Im Fïhiahr 1917 nochmaliger Ruhrrück fall. Lazarcttbehandlung von einem Mon:t Diluer: nochmals cin starker Rückfall im Aupust 1918. Während des Jahres 1919 hat cr wechscind Durchfälle und Verstopfung gehabt, bei den Durchfällen ist ilım oft ter Blutgehilt wicder aufgefallen. Seit Ende 1918 hat sich in seincm Allgemeinbefinden zicm. lich plötzlich cine Veränderung gezeigt. insofcm der sonst ruhige und gelassene Малn schr rcizbar und hälfig wegen plötzlich auftrctender Anfälle von "Herzkrämpfen" im Schlaf gestört wird und daB cr be ganz kleinen Anstrengungen unverhältnismäßig stark schwiłzt. Aus $\mathrm{dem}$ Befund ist hervorzulıcben. daß es sich um cinen äıßerlich kräftig gebauten Menschen handelt mit gut entwickelter Muskulatur. und Feftpolster. Dic Schilddrüsc ist gut fühlbar, aber nicht vergrößcrt. Seh starke flächenförmigc Dermorraphie und schr erhcbliche Schweiß. ausbrüche bei lcichter scclischer Errcgung werden bei der crsten Untersuchung schon festgestellt. Löwvsche Reaktion ncrativ. Am Herzen normale Verhältnisse. der Puls schwankt zwischen 80 und 100 Schlägen in der Minute. Blutdruck $100 \mathrm{~mm}$ Hg. Probefrühstück: $70 \mathrm{ccm}$, freie Salzsäurc 5. Gesamtsäıre 25. Rektoskonic: schon nach $10-12 \mathrm{~cm}$ sehr starke Spasmell, die ein weiteres Vordringen reihindern: bis dahin ist die. Darmschlcimhaut stark merötet und glasig geschwollen. von durchsichtigem Schleim hier und da bcdcckt. kein Geschwïr bis in diesc Höhe. Während der Beobachtung werden täglich 2-4 Stühle abgesctzt, die meist mittelbreiig, von dunkelbrauner

1) Vgl. dazu das Referat von H. StrauB uber die Polgezustănde der Ruhí auf der Homburger Tagung (erscheint im Arch. f. Verdauungskr.). in dem der Autor Thyreotoxikosen-bei postdysenterischer Kolitis ebenfalls erwahnt. 
Farbe sind und neutrale Reaktion aufweisen. Makroskopisch deutlich Bindegewebsreste, zièmlich viel feiner und oft grobflockiger Schleim. In mehreren Stühlen sehr große Mengen von schlecht verdautell Muskelfasern mit erhaltener Querstreifung und scharfen Kanten. Keine Vermehrung von Stärkeresten und Fett bei Probediät.

Der Blutzucker beträgt niichtern $0,122 \mathrm{~g} 0 \%$. Nach Zufulır von $300 \mathrm{~g}$ 'Brot in den ersten 2 . Stunden in Urin Nylander negativ, nach 4 Stunden schwach positiv, nach 6 St tmiden deutlich positiv. Blutbild: RBK. 4380000 , WBK. 7200 . Eosinophilie von. $10 \%$.

Die psychische Erregung des. Mannes war augenscheinlich; so hat er nach der Rektoskopie, die ihm nach seiner eigenen Angabe nicht wesentliche Schmerzen bereitet Juit, laltlos eine halbe Stunde lang geschluchat. Auch der Schlaf war, wie die Beobachtung zeigte, nur sehr wenig tief.

Dieses Krankheitshild ist mit einigen Modifikationen nicht selten anzutreffen. Ich schätze, daß man einen melır oder weniger deutlichen thyreotoxischen Symptomenkomplex im angedenteten Sinne bei etwa jedem 5.-6. Falle von chronischer postdysenterisclier Kolitis antreffen wird. Lo e we nthal (5) etwähnt in seiner Arbeit über das vorliegende Krankheitsbild diese Komplikation nicht. Einen Hinweis auf eine scholl von anderer Seite für möglicl gehaltene innere Verbindung von Dickdarm und Schilddrüse wäre in einem Zitat in $\mathrm{B}$ ied Is Werk zu erblicken, wonach Hemmete $\mathrm{H}$ die Entstehung ciner echten Basedowscheıi Erkrankung auf eine-chronische Kolitis zurückführt. -Die Originalmitteillung Hemmeters ist mir zur Zeit nicht zugäuglich.

Diese Fälle brauchen durchaus nicht bis zum voll ausgeprägten Bild der Basedowschen' Erkrankung zu kommen, wenigstens soweit meine bisherigen. Erfahrungen reichen. Fast alle bekannten Symptome der leichten Schilddrüsenvergiftung können in wechselnder Kombiıntion vorkommen. Eines aber, auf das im allgemeinen nicht der Wert gelegt zu sein scheint, wie es das.verdient, habe ich bei allen diesen Fällen in deitlicher Ausbildung gefunden, das ist die ve fmehrte Zuckerausscheidung nach Phlorizin-Injektion. Dieses Alkaloid mach't bekanntermaßen eine. Zuckerausscheidung. im Urin ohne Steigerung des Blutzuckers. Die allgcmeine Ansicht geht dahill, da $\mathrm{B}$ die Nierenzelle so beeinflußt 'wird, daß sie entweder den vorhandenen Blutzucker filtricrt oder aber auch aktiv Zucker : lus Material bildet, das im Blıt nicht als Zucker kreist. Von der Nierenwirkuing unabhängige Störungen anzunehmen, dazu lag bislang ansclıeinend keine Nötigung vor. Doch sind schon Bedingungen aufgedeckt worden die auf die Größe der Glukosurie einen maßgebenden Einfluß auśliben. $\mathrm{C}$ a ro (6) hat anschicinend zuerst gefunden, $\mathrm{da} \beta$ die Basedowsche Erkrankung die Zuckerausscheidung erheblich vermehrt und daß auch die thyreotoxischen Zustände, besonders auch die Gravidität, in - gleichem Sinne von Einfliiß sind. Er fand, daß besonders dic prozentuale Ausscheidungskurve stärkẹ ansteigt als bei normalen Menschen und d:ı fi Fälle mit alimentärer Glukosuric anch eine vermshrte Phlorizinglukosuric aufweisen. " $\mathrm{Coh} n$ und $P \mathrm{Pe}$ iser (7) haben später "dieses Symptom benutzt, um bei-otganjschen Pankreaserkrankungen eine : thyreotische Mitbeteiligung festzusstellen, was ihnen :uch in 5 Fälleıl gelungen ist. Ich habe den Eindruck, da $\beta$ die vermehrte Phlorizinglukosüirie für die Aufdecking dieser Schädigungen sehr gute. Dienste leisten kann. Die thyreotoxischen Fälle von chronischer Kolitis haben sämitlich in diesem Sinne positiv reagiert. Wenn wir dem gesunden Menschen eine' !lalbe Stunde nach der Mittagsmahizeit $0,01 \mathrm{~g}$ Phlorizin intramuskulär injizieren, so werden im Durchschuitt innerhalb der'nächsten 6 Stunden maximal etwa $6 \mathrm{~g}$ Zucker ausgeschieden. In unsereı Fällen wurde der Úrin zweistündfich gesammelt und kontrolliert. Bei thyreotoxisclıen Zuständen vermehrte sich diese Menge auf das 2-3 faclie. In einem Falle, der bis jetzt die stärkste Reaktion gah, betrug die Gesamtmenge des ansgeschiedenen Zuckers $23.7 \mathrm{~g}$. Dieser Fall zeigte neben seiner clironisclien Kolitis ausgesprochenen Fingertremor, profuse Sclıveiße, Anfälle von Tachyk:ırdie, Dermographismus und zweifellose Vergrößerung der Schilddrüse; Löwy war negativ. Einer der Kontrollfälle, bei dem es sich um eine seit langein. bestcheride Akromegalie mit röntgenologisch nachweisbarer erheblicher Vergrößerung des Türkensattels handelte, der ancli gleichzeitig durch Veränderungen der Haut und der Haare auf cinen myxödematösen Einschlag scliließen ließ, ergah der Phlorizinversuch nur eine Gesamtausschıeidung von $0,7 \mathrm{~g}$.Zucker. Der Gesunde scheidet zum mindesten etwa $2 \mathrm{~g}$ aus.

Hat nun fraglos diese Beobachtung auch vom rein klinisch-therapeutischell Standpunkt aus ilire - Bedeutung, insofern dic Behandlung weitgehende Rücksicht auf die ínkretorische Erkrinkung vehmen muß, so möchte ich doch noch auf zurr Zeit zivar noch spekulative, aber für die Zukunft nicht :mlnwichtige Ausblicke hinweisen. Weın auf irgendeine Gruppe von Krankheitszuständen, so paßt auf diese sekundären endokrinen Störungen der $M$ artiussche Begriff ,erworbener Konstitutionalismus". (8). Der Mensch erfährt in seiner G̈esanıtverfassung eiñe Umwandlıng derart, daß der ganze Körper' neue, inn zwar schädliche, vom Gesunden abweichende Eigenschaften erhïlt, die sich in einer gegen, die Norm verschobenen Reaktionsfähigkeit auf äußere und innere Reize ausspricht. Daß manche unserer Fälle auf der Grenze der Basedowschen Erkrankung. stehen, ist nach dem Dargelegten ohne weiteres verständlich. Sie gewinnen also eine neuartige Krankheitsdisposition hinzu, die sie voraussichtlich ohne die konditionelle Darmkrankheit nicht erworben haben würden. Welche und wie erhebliche Reize noch hinzukommen müssen, um aus diesen sekundär thyreotischen Darmkranken echte Basedowkranke zu machen, muß die Erfahrung zéigen. Nach meinen Fällen kanı ich mich des - Eindrucks nicht erwehren; daß der thyreotische Vergiftungszustand oft nicht als ganz leicht zu beurteilen ist und auch dem Anschein nach einen fortschreitenden Charakter aufweist.

Dieser Vorgang der sekundären Aenderung der gesamten Körperverfassung auf Grund einer bestimmten inkretorischen Störung hat aber wohl noch eine Bedeutung, die über den betroffenen Menischen selber hinausgelıt. Wir wissen, daß besondérs der Krankheitskomplex des Basedow (vornchmlich beim männlichen Geschlecht) cine ausgesprochene direkte Erblichkeit zeigt. Den ganzen Einfluß inkretorischer Störungen auf das Keimplasma lıaben wir erst in der neuestén Zeit verstehen lernen, nachdem die Untersuchungen von Gunder. natsch, Adler, Abderhalden und namentlich $\mathrm{Hart} \cdot(9)$ die Wirkungen von Schilddrüse und Thymus auf das Wachstum und die Metamorphose yon Kalthlütern gezcigt haben. Es sei davon hier nur so viel erwähnt, daß die Drüsen mit innerer Sekretion augenscliein. lich die vermittelnden Organe darstellen zwischen der Kontdition oder den Individualwirkungen der Außenbedingungen urtd der Konstitution, dem Keimplasma. Es scheint das alte Problem der Vererbung erworbener. Eigenschaften auf diesem Wege einer Lösung; und zwar in positivem Sinne, entgregengeführt zal werden. Unter dieșem Giesichtswinkel gewinnen Beobachtungen sekundärer inkretorischer Störnngen bei organischen Erkrankungen eine besondere Bedeittung. Die Vorstellung ist zum mindesten erwägenswert ob nicht ein solcher inkreto. rischer Einschlag des Kranklıeitsbildes auf das Keimplasma gleichsinnig oder auch irgendwie anders schädigend einwirken kann, und dah wir gerade in Fällen der geschilderten Art eine Generation vor uns lıaben, deren Deszendenz hinsichtlich ihre's Darmkanils konstitutionell gef:ïhrdet erscheint. Das. Jäßt sich natìrlich -jetzt niclıt beweisen, ist aber ein Gedankeng:ang von prinzipieller Wichtigkeit. Die auffällige familiäre Häufung von Darmerkrankungen - ich denke nur an das Magengeschwür, für das Spiegel (10) eine Vererbung in über $60 \%$ der Fälle ermittelt hat, weiter auch an den Magenkrebs und die gar nicht selten familiär auftretendén Dyspepsien u. a. - ist jedem Arzt geTäufig. Irgendeine brauchbare Vorstelling darüber, wie etwa in cine Deszendenterrreihe einc solche Organbelastung hineinkommt, haben wir nicht. Dic augenscheinlich von einer-Mehrzahl von Autoren geteilte Ansicht über die Entstehıng einer Kranklıeitsanlayge geht dahin, daß es sich hierbei um einen Vorgang handeln müsse, der dem von der experimentellen Vererbungslehre lier geläufigen Mutationsprozeß ähnliclı jst oder ihm-genall-entspreche. (vgl. Na egeli [11], D resel [12]). Doch müssen wir uns klarmachen, daB mit der Aufstellnng des Begriffs derMutation für eine Krankheitsentstehung noch nicht viel mehr gewonnen ist als eine Bezeichulung. Wir wissen zwar daß eine Reihe von äußeren Faktoren bei gewissen Tierarten dauernd vererbbare Mutationen hervorbringen kann, doch ist uns die besondere Art und Weise ihres Zustandekommens, der innere $\mathrm{Fak}$ tor dabei, noch völlig. dunkel. Haecker (13) hat einen Weg gezeigt, auf dem es vielleicht möglich ist, den inneren Faktoren näher 7.u kommen. Seine entwicklingsgeschichtliche Eigenschaftsanalyse (Phänogenetik) setzt sich zum Ziel, die Ontogenese eines in irgendeiner Richtung abweichenden Organismus bis auf den Punkt zurückzuverfolgen, in dem sich diese Abweichung zum ersten Male bemerk bar macht. H Hecker nenut diesen Zeitpunkt, in dem also irgeridwelche Einflüsse cine von der Regel abweicliende Entwicklung herbei7.uführen beginnen. dic phänokritische Phase. Ueber diesen Zeitpunkt hinaus muß die phänokritische Ursache gesucht werden, die den Ansto $B$ zu der abweichenden Entwicklung der fertigen. Außeneigenschaft abgibt. Unter dem Einfluß der Untersuchungen der oben genannten Aıtoreı ist man versucht, der Einwirkung inkretorischer Drüsen einen wesentlichen Anteil an diesen phänokritischen Ursachen zuzusprechen. - Aendern inkretorische Einflüsse in der Tat das Keimplasma im Sinne irgendeiner beim Deszendenten auftretenden. Organanomalie, so müB ten wir diese Einflüsse als die phänokritische Ursache des Auftretens einer. Krankheitsanlage anerkeninen und würden đamit wesentlict den formell scharf umschriebenen, aber genetisch schr schwer verständlicheıl Vorgang der Mutation aufklären.

Dic Möglichkeit, daß ein solcher inkretorischer Einfluß auf das Keimplasma in der angedeuteten Weise zustandekommt, ist jedenfalls im Auge zu behalten. Selbstverständlich wird es nicht immer die Schilddrüse scin; dic für dic Störung verantwortlich ist, in unseren Fälleı steht ilıre Schädigung klinischı nur sehr greifbar im. Vordergrund. Sekundäre Störungen anderer inkretorischer Drüsen sind bekauntlich oft nur vermutungsiveise festzıstellen; wenn wir dabei von ganz. aus geprâgten Kraukheitsbildern (Akromegalie, Addison) absehen. Derartigen Fragen müßte aber in Zukınft ein größeres Interesse entgevengebracht werden. Namentlich wird das katamnestische Studium dieser Fälle und die Untersuchung ihrer Deszendenten eine wichtige Aufgabe darstellen, für die die Beob:ichtung sekundärer inkretorischer Erkrankungen die Grundlage abgibt.

1. M. m. W. 591912 S. $2669-2$. Arch. f. Verdauungskr. $20 \mathrm{~S} .1 .-3$. D. m. W. $1915 \mathrm{Nr} .34 .-4$. Magenkrankheiten durch Kriegseinwirkungen 1919 S. $27 \mathrm{ff}$ - - 5. Arch. Verdauungskr. 25 S. 465 - 6. Mitt. Grenzgeb. 23 H. 1 S. 100. - 7. D. m. w. 1912 iNr. 2 S. 60. - 8. Konstitution und Vererbung 1914 S. $40 \mathrm{ff}$ - 9 . Zschr. f. angw. Anat. u. Konstit. 6 S. 71 -10 . D. Arch. f. klln. M. 126 S. 45. - 11. Zschr. f. angew. Arat. u. Konstit 6 S. 33. - 12. VIrch. Arch. 224 S. 256. - 13. Entwicklungsgeschichtliche Eigenschaftsanalyse (Phünogenetik) $1018 \mathrm{~S} .5 \mathrm{ft}$ 